\title{
THE ANALYSIS OF MULTIPLE LINKAGE DATA
}

\author{
A. R. G. OWEN \\ Department of Genetics, Cambridge
}

Received 22.xi.52

IN these notes $I$ indicate methods applicable to the interpretation of multiple linkage tests involving three or more linked loci. The methods advocated are based on two extensions of Kosambi's simple and extremely useful theory of linkage relations, the purpose of the extensions being to widen the range of usefulness without losing the powerful simplicity of that theory.

In the first place Kosambi's theory (I) may be supplemented by the introduction of a quantity $\mathrm{K}$ whose value varies with position on the chromosome arm. I have shown elsewhere that we may expect high values of $\mathrm{K}$ to be realised for sets of loci in the neighbourhood of the centromere, and low values to occur near the termini of the chromosome, $\mathrm{K}$ taking the value unity at some point related to the middle of the arm. In a survey (2) of data on Chromosomes II and III of Drosophila melanogaster (not yet fully published), I found excellent agreement with these predictions. Thus it seems likely that in other organisms the value of $\mathrm{K}$ as derived from experiments on multiple crossing-over can be of some use in suggesting the location on the chromosome arm of the loci concerned. In Part I, I deal with the statistical problems involved in the estimation of $\mathrm{K}$ and of the trend of $\mathrm{K}$ in the cases of three and four linked loci, and discuss some actual data obtained for the Agouti-pallid region of Chromosome $\mathrm{V}$ in the mouse.

Part II is of more abstract interest and extends Kosambi's theory (without introducing $\mathrm{K}$ ) to multiple recombination with as many as six linked loci. Here the mathematical method used is not identical with that of Kosambi but proceeds from the general theory of linkage relations developed by Sir Ronald Fisher and myself (2-7).

Part III introduces $\mathrm{K}$ into Kosambi's theory and gives an elementary way of generalising Kosambi's formulæ to the special but useful cases of several fairly closely linked loci such as are treated in I. 4 .

\section{ESTIMATION OF THE KOSAMBI COEFFICIENT}

\section{INTRODUCTION}

The Kosambi coefficient, denoted by $\mathrm{K}$, is defined (5) in terms of the recombination frequencies of 3 linked loci, $A, B$ and $D$ by means of the equations

$$
\mathrm{K}=\frac{y_{1}+y_{2}-y_{1+2}}{4 y_{1} y_{2} y_{1+2}}=\frac{y_{12}}{2 y_{1} y_{2} y_{1+2}} .
$$

Here the suffixes $I$ and 2 refer to the segments $A B$ and $B D$, while the 
suffix $I+2$ refers to the combined segment AD. Thus any three loci are characterised by a value of $\mathrm{K}$. It will be seen that $\mathrm{K}$ is equal to $\mathrm{C} / 2 y_{1+2}$, where $\mathrm{C}$ is the genetical coincidence for the two segments, defined by Stevens (8) as $y_{12} / y_{1} y_{2}$. Kosambi ( $\mathrm{I}$ ) obtained the addition formula

$$
y_{1+2}=\frac{y_{1}+y_{2}}{\mathrm{I}+4 y_{1} y_{2}}
$$

by an assumption equivalent to setting $\mathrm{C}$ equal to $2 y_{1+2}$; i.e. to taking the value of $\mathrm{K}$ as unity. Regions of the chromosome in which $\mathrm{K}$ is not significantly different from unity have been called Kosambi regions (5), and in such regions interference is said to be at the Kosambi level $(5)$. I have given $(2,5)$ theoretical reasons for supposing that in regions near the centromere $\mathrm{K}$ has a high value greater than unity, and diminishes steadily with increasing distance from the centromere, becoming relatively small near the terminus of an arm. Consequently there is a Kosambi region, where $\mathrm{K}=\mathrm{I}$, situated medianly on the arm.

These expectations have been well fulfilled in the case of Chromosomes II and III in Drosophila melanogaster (2). Therefore, in the absence of evidence to the contrary, it seems reasonable in other organisms to use the observed $\mathrm{K}$ values as providing some indication of the distance of the loci concerned from the centromere, and of the orientation of segments relative to the centromere. This note is concerned with estimation of $\mathrm{K}$ values when data on 3 or 4 loci are available.

\section{THE USE OF THREE POINT DATA}

Backcross experiments provide the best determinations of the recombination fractions, and therefore of $G$ and $K$. In such an experiment involving two segments $\mathrm{AB}$ and $\mathrm{BD}$, suppose that out of a total of $n$ organisms bred the observed class frequencies are

$\begin{array}{llll}0 & 1 & 2 & 12 \\ a_{0} & a_{1} & a_{2} & a_{12}\end{array}$

Here $a_{0}$ show recombination in neither I nor 2, $a_{1}$ show recombination in $\mathrm{r}$ but not in 2, $a_{2}$ show recombination in 2 but not in $\mathrm{I}$, while $a_{12}$ show simultaneous recombination in $\mathrm{I}$ and 2. Thus

$n_{1}=a_{1}+a_{12}$ show recombination in $\mathrm{r}$,

$n_{2}=a_{2}+a_{12}$ show recombination in 2 ,

$n_{1+2}=a_{1}+a_{2}$ show recombination in the combined segment $\mathrm{I}+2(\mathrm{AD})$

$n_{12}=a_{12}$ show simultaneous recombination in $\mathrm{I}$ and 2.

The estimators of $y_{1}, y_{2}, y_{1+2}, y_{12}$ are

$$
\hat{y}_{1}=n_{1} / n, \hat{y}_{2}=n_{2} / n, \hat{y}_{1+2}=n_{1+2} / n, \hat{y}_{12}=n_{12} / n \text {. }
$$

The sampling variance of any such function is $\mathrm{V}(y)=y(\mathrm{I}-y) / n$. 
The estimator of $\mathrm{C}$ is $\hat{\mathrm{C}}=n n_{12} / n_{1} n_{2}$, and Stevens (loc. cit.) has shown that its sampling variance is

$$
\mathrm{V}(\hat{\mathrm{C}})=\mathrm{C}\left(\mathrm{I}-\mathrm{C} y_{1}-\mathrm{C} y_{2}-\mathrm{C} y_{1} y_{2}+2 \mathrm{C}^{2} y_{1} y_{2}\right) / n y_{1} y_{2} \text {. }
$$

This may be estimated by inserting the estimates $\hat{y}_{1}, \ldots, \hat{\mathrm{C}}$, and a crude test of the significance of the difference of $\mathrm{C}$ from unity (i.e. a test for the presence of genetical interference) may be based on the estimated standard error or on the variance. E.g. with the data

$$
\begin{array}{ccccc}
\text { o } & \text { I } & 2 & \text { I } 2 & n \\
\text { II } 6 & \text { I } 82 & 375 & 24 & \text { I } 697
\end{array}
$$

we have the percentage values

$$
\hat{y}_{1}=\mathrm{I} 2 \cdot \mathrm{I} 39, \quad \hat{y}_{2}=23.5 \mathrm{I} 2, \quad \hat{\mathrm{c}}=49.55^{\mathrm{I}}
$$

Estimated $\mathrm{V}(\hat{\mathrm{C}})=84 \cdot 2 \mathrm{I} 76$ (per cent.) ${ }^{2}=0 \cdot 008,42 \mathrm{I}, 76$

Estimated $s(\hat{\mathrm{C}})=9 \cdot 1770$ per cent.

Thus $\hat{\mathrm{C}}=49 \cdot 55 \pm 9 \cdot 18$ per cent. which is significantly less than unity and indicative of genetical interference.

The maximum likelihood estimator of $\mathrm{K}$ is simply

$$
\hat{\mathrm{K}}=\hat{\mathrm{C}} / 2 \hat{y}_{1+2}=n^{2} n_{12} / 2 n_{1} n_{2} n_{1+2} \text {. }
$$

This statistic is biassed, the bias being given approximately by

$$
\beta=\hat{\mathrm{K}}\left(\frac{3}{n_{1+2}}-\frac{3}{n}-\frac{4 \hat{\mathrm{K}} n_{12}}{n^{2}}\right)
$$

The estimated sampling variance is given by

$$
\mathrm{V}(\mathrm{K})=\mathrm{K}^{2}\left\{\frac{\mathrm{I}}{n_{12}}+\frac{5}{n_{1+2}}+\frac{2 n_{12}}{n_{1} n_{2}}-\frac{\mathrm{I}}{n_{1}}-\frac{\mathrm{I}}{n_{2}}-\frac{4}{n}-\frac{4}{\frac{\left(n_{1}+n_{2}\right) \hat{\mathrm{K}}}{n^{2}}}\right\}
$$

or may be got by inserting the estimates in

$$
\frac{\mathrm{K}^{2}}{n}\left\{\frac{\mathrm{I}}{y_{12}}+\frac{5}{y_{1+2}}+{ }_{2} \mathrm{C}-\frac{\mathrm{I}}{y_{1}}-\frac{\mathrm{I}}{y_{2}}-4-4 \mathrm{~K}\left(y_{1}+y_{2}\right)\right\} .
$$

In the example we have

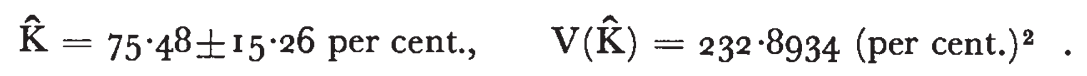

The bias is not seriously large, since we have

$$
\beta=0.004,04 \mathrm{I}=0.40 \text { per cent. }
$$

To test departure of $\mathrm{K}$ from unity we calculate

$$
\chi^{2}{ }_{[1]}=(\hat{\mathrm{K}}-\mathrm{I})^{2} / \mathrm{V}(\mathrm{K})=4.53 \mathrm{I} 7,
$$

where $\mathrm{V}(\mathrm{K})$ is now calculated from equation ( $\mathrm{I}$ ) with $\mathrm{K}$ put equal to unity.

$\mathrm{K}$ therefore deviates just significantly from unity. 
In cases such as the present example when $\mathrm{K}$ is not very significantly different from unity a more refined procedure ought next to be used. We assume $y_{1}, y_{2}$ as free parameters and take $y_{1+2}, y_{12}$ as given as functions of $y_{1}, y_{2}$ by Kosambi's formulæ. A maximum likelihood scoring technique gives amended estimates which are then used to calculate expected class frequencies for a $\chi_{(1)}^{2}$ fit to the observations. If the fit is good, i.e. if $\chi^{2}$ is not significantly large then we may retain the hypothesis $K=\mathbf{I}$. If the fit is bad we may then estimate $K$ as above, together with its estimated sampling variance and standard error.

\section{THE USE OF FOUR POINT DATA}

When data on four loci A, B, D, E are available, the appropriate treatment is determined by the closeness of the observed linkage between them. When the segments are sufficiently long for doubles to be at least moderately frequent, the best use of the data requires that $\mathrm{K}$ be calculated separately for the two triads $\mathrm{ABD}, \mathrm{BDE}$, the data being reduced to three point data in respect of each triad. If the values $K_{1}, K_{2}$ are significantly different then the direction of the trend may be taken as presumptive evidence of the orientation of the segment $\mathrm{AE}$ relative to the centromere O. E.g. if $\mathrm{K}_{1}>\mathrm{K}_{2}$ the preferred order is OAE.

To test the significance of the difference $\left(\mathrm{K}_{1}-\mathrm{K}_{2}\right)$ we may compare it with its sampling standard deviation using either a $\chi$-, $\chi^{2}$ - or $t$-test. The variance of $\left(\mathrm{K}_{1}-\mathrm{K}_{2}\right)$ is

$$
\mathrm{V}\left(\mathrm{K}_{1}\right)-{ }_{2} \mathrm{C}\left(\mathrm{K}_{1}, \mathrm{~K}_{2}\right)+\mathrm{V}\left(\mathrm{K}_{2}\right),
$$

where $\mathrm{C}\left(\mathrm{K}_{1}, \mathrm{~K}_{2}\right)$ is the sampling covariance of $\mathrm{K}_{1}$ and $\mathrm{K}_{2}$, given approximately by

$$
\begin{aligned}
\hat{\mathrm{K}}_{1} \hat{\mathrm{K}}_{2}\left(\frac{n_{12}}{n_{1} n_{2}}+\frac{n_{23}}{n_{2} n_{3}}+\frac{n_{13}}{n_{1} n_{3}}+\frac{a_{2}}{n_{2} n_{1+2}}+\frac{a_{2}}{n_{2} n_{2+3}}+\frac{a_{2}}{n_{1+2} n_{2+3}}\right. \\
\left.+\frac{n_{123}}{n_{12} n_{23}}-\frac{n_{123}}{n_{1} n_{23}}-\frac{n_{123}}{n_{3} n_{12}}-\frac{1}{n_{2}}-\frac{4}{n}\right)
\end{aligned}
$$

This expression has been derived by means of a formula cognate to that given by Sir Ronald Fisher for the approximate sampling variance of a statistic (9). If $m_{1}, m_{2}, \ldots, m_{r}$ (totalling $n$ ) are the frequencies observed in $r$ classes subject to a multinomial distribution, and if $\mathrm{T}_{1}, \mathrm{~T}_{2}$ are homogeneous functions of degree $\mathrm{o}$ or $\mathrm{I}$ in $m_{1}, m_{2}, \ldots$. , $m_{r}$ and $n$, then we have as the approximate sampling covariance of $\mathrm{T}_{1}$ and $\mathrm{T}_{2}$ the expression

$$
\mathrm{C}\left(\mathrm{T}_{1}, \mathrm{~T}_{2}\right)=\sum_{i=1}^{r} m_{i} \frac{\partial \mathrm{T}_{1}}{\partial m_{i}} \frac{\partial \mathrm{T}_{2}}{\partial m_{i}}-n \frac{\partial \mathrm{T}_{1}}{\partial n} \frac{\partial \mathrm{T}_{2}}{\partial n} .
$$


When $T_{1}=T_{2}$, this becomes Fisher's formula

$$
\mathrm{V}(\mathrm{T})=\Sigma m\left(\frac{\partial \mathrm{T}}{\partial m}\right)^{2}-n\left(\frac{\partial \mathrm{T}}{\partial n}\right)^{2}
$$

Each formula holds exactly when $T_{1}$ and $T_{2}$ are linear functions of $m_{1} / n, \ldots ., m_{r} / n$.

\section{THE USE OF FOUR POINT DATA FOR SHORT SEGMENTS}

When $A$ and $E$ are close together $K_{1}$ and $K_{2}$ will differ significantly. Double recombination classes will occur with small frequencies and triples will be absent. For if $p, q, r$ are the three recombination fractions the expected frequencies of doubles and triples will be given to good approximation by the expressions given below (part III), namely :-

$$
\begin{gathered}
y_{12}={ }_{2} \mathrm{~K} p q(p+q), \quad y_{23}={ }_{2} \mathrm{~K} q r(q+r), \quad y_{13}=2 \mathrm{~K} p r(p+2 q+r), \\
y_{123}={ }_{4} \mathrm{~K}^{3} p q r\left\{(p+q)(q+r)-\frac{1}{3} q^{2}\right\} .
\end{gathered}
$$

If the observations are

$$
\begin{array}{ccccccccc}
\mathrm{o} & \mathrm{I} & 2 & 3 & 23 & \mathrm{I} 3 & \mathrm{I} 2 & \mathrm{I} 23 & \text { Total } \\
a_{0} & a_{1} & a_{2} & a_{3} & a_{23} & a_{13} & a_{12} & 0 & n
\end{array}
$$

then it may be shown by inspection of the maximum likelihood equations for the four unknowns $p, q, r, \mathrm{~K}$ subject to joint estimation, that the estimators approximate closely to the "intuitive" ones $\hat{p}=\left(a_{1}+a_{12}+a_{13}\right) / n$, etc. $\hat{\mathrm{K}}=\left(a_{23}+a_{13}+a_{12}\right) / \mathbf{D}=$ (observed doubles) $/$ (expected doubles).

Here $D$ is the expected total frequency of doubles calculated as

$$
\begin{aligned}
\mathrm{D} & =n\left(y_{12}+y_{23}+y_{13}\right) \\
& =\frac{2}{3}(p+q+r)^{3}-\frac{2}{3}\left(p^{3}+q^{3}+r^{3}\right)
\end{aligned}
$$

estimated by inserting $\hat{p}, \hat{q}, \hat{r}$. Without serious error we may for this purpose take for $\hat{p}, \hat{q}, \hat{r}$ the quantities $a_{1} / n, a_{2} / n, a_{3} / n$. Thus we get the approximate estimator

$$
\hat{\mathrm{K}}=3^{n^{2}\left(a_{23}+a_{13}+a_{12}\right) / 2\left(s_{1}{ }^{3}-s_{3}\right)}
$$

where $\quad s_{r}=a_{1}{ }^{r}+a_{2}{ }^{r}+a_{3}{ }^{r}, \quad(r=1,2,3, \ldots)$.

For the variance of $\mathrm{K}$, we get by the approximate method of the last section

$$
\mathrm{V}(\hat{\mathrm{K}})=\hat{\mathrm{K}}^{2}\left\{\frac{\mathrm{I}}{a_{23}+a_{13}+a_{12}}+\frac{9\left(s_{1}{ }^{5}-2 s_{1}{ }^{2} s_{3}+s^{5}\right.}{\left(s_{1}{ }^{3}-s_{3}\right)^{2}}-\frac{4}{n}\right\}
$$

The first term is usually dominant so that to a rough approximation

$$
\mathrm{V}(\hat{\mathrm{K}})=\hat{\mathrm{K}}^{2} / \text { (observed doubles). }
$$


Example.-In a four point backcross experiment recently completed, of which a full account is not yet published, the four loci Agouti, undulated, wellhaarig, and pallid in the house mouse were employed. The following class frequencies were obtained.

\begin{tabular}{|c|c|c|c|c|c|c|c|c|}
\hline & 1 & 2 & 3 & 23 & 13 & 12 & 123 & $n$ \\
\hline $\begin{array}{c}\text { From female heterozygotes } \\
\text { From male heterozygotes } \\
\text { Total }\end{array}$ & 117 & 185 & 104 & 1 & 0 & 0 & 0 & 2503 \\
115 & 111 & 56 & 1 & 0 & 1 & 0 & 2499 \\
\hline & 232 & 296 & 160 & 2 & 0 & 1 & 0 & 5002 \\
\hline
\end{tabular}

On the combined data for males and females we have

$$
\hat{p}=4.66 \text { per cent., } \hat{q}=5.98 \text { per cent., } \hat{r}=3.24 \text { per cent. }
$$

Observed doubles $=3 . \quad s_{1}=688, s_{1}{ }^{2}=47 \cdot 3344 \times 10^{4}$

$$
\begin{array}{ll}
s_{3}=42.5175 \times 10^{6}, & s_{1}^{3}=325.6607 \times 10^{6}, \\
s_{5}=304.9229 \times 10^{10}, & s_{1}^{5}=1.5415 \times 10^{14}
\end{array}
$$

Thus $\hat{\mathrm{K}}=39 \cdot 76 \pm 23 \cdot 1105$ per cent., $\mathrm{V}(\hat{\mathrm{K}})=0.055,263$, and $\hat{\mathrm{K}}$ is significantly less than unity, its value being suggestive of a region towards the chromosome terminus or at least between mid-arm and the terminus. The segment Agouti-pallid is thus between $30-100 \mathrm{cM}$ from the centromere according as to what is assumed for the map length of the arm.

The treatment is however rather inaccurate on account of the marked sex-difference of two of the recombination fractions, and is best carried out separately for the two sexes. We obtain

\begin{tabular}{|lc|c|c|}
\hline & & K per cent. & Information \\
\cline { 3 - 4 } & & & \\
\hline Females : &. & $\cdot$ & $16 \cdot 24 \pm 16 \cdot 41$ \\
Males & $96 \cdot 63 \pm 68 \cdot 29$ & $37 \cdot 15$ \\
Combined & $\cdot$ & $\cdot$ & $2 \cdot 30$ \\
$20 \cdot 92 \pm 15 \cdot 09$ & $39 \cdot 45$ \\
\hline
\end{tabular}

Thus in the females $\mathrm{K}$ is significantly low, but in the males does not differ significantly from o or unity. On the basis of the male and female data considered jointly we might legitimately assume the segment to be moderately distant from the centromere, since the difference between male and female $K$ values is not itself significant.

\section{SUMMARY}

Methods of estimation are given for $\mathrm{K}$ and its sampling variance in the case of triads of loci, and for the covariance of $K$ values in overlapping triads. It is shown how to estimate $\mathrm{K}$ in the case of three short segments with special reference to the Agouti-pallid region of Chromosome $\mathrm{V}$ in the house mouse. It is suggested that Agouti is moderately far from the centromere. 


\section{THE FREQUENCIES OF SIMULTANEOUS RECOMBINATION OF SEVERAL LINKED LOCI}

\section{INTRODUCTION}

Kosambi (I) proposed the formula

$$
y=\frac{1}{2} \tanh 2 x
$$

as an approximate representation of the relation between the genetical map-length $x$ of the segment joining two loci and $y$ the amount of genetical recombination between those loci. For 3 loci defining adjacent segments $x_{1}$ and $x_{2}$ this formula implies that

$$
y_{12}=2 y_{1} y_{2} y_{1+2}=2 y_{1} y_{2} \frac{y_{1}+y_{2}}{1+4 y_{1} y_{2}} \text {, }
$$

where $y_{12}$ is the frequency of recombination in both simultaneously, and $y_{1+2}$ is the frequency of recombination over the combined segment $x_{1}+x_{2}$. I have indicated $(5,7)$ the conditions under which these approximations are valid. If, in particular, the interference is represented by the function $4 u \exp (-2 u)$ employed in previous work (4-7), then, for a segment near the (metrical) mid-point of a long chromosome arm, we obtain the relation

$$
y=\frac{1}{2}\left(\mathrm{I}-e^{-2 x} \cos 2 x\right) .
$$

For segments less than $35 c \mathrm{M}$ in map-length this function differs from $\frac{1}{2} \tanh 2 x$ to an extent which in practice is quite inappreciable, the values of $y$ given by the two formulæ being discrepant by at most 8 parts in 302. Thus Kosambi's formula receives a simple theoretical justification as an approximation likely to apply in segments median to long chromosome arms.

Using the methods indicated by previous work it has been shown (7) that there are simple expressions giving all recombination frequencies for 4 loci ( 3 segments) which also are likely to apply when all 4 loci are reasonably close to metrical mid arm. I gave the new relations

and

$$
y_{13}=y_{1} y_{3}-z_{1} z_{3} e^{-4 x_{2}}, \quad w_{12}=w_{1} w_{2}
$$

Here $\quad z=\frac{1}{2} e^{-2 x} \sin 2 x, \quad w=y+i z, \quad i=\sqrt{-\mathrm{I}}$,

and $\quad \mathrm{R}\left(w_{1} w_{2} w_{3}\right)=y_{1} y_{2} y_{3}-y_{1} z_{2} z_{3}-y_{2} z_{1} z_{3}-y_{3} z_{1} z_{2}$.

I showed further that these relations approximate to

and

$$
y_{13}=y_{1} y_{3}\left\{\mathrm{I}-\frac{\mathrm{I}-2 y_{2}}{\mathrm{I}+2 y_{2}} \frac{\left(\mathrm{I}-2 y_{1}\right)\left(\mathrm{I}-2 y_{3}\right)}{\mathrm{I}+4 y_{1} y_{3}}\right\}
$$

These equations may be regarded as natural generalisations of Kosambi's formulæ. While receiving their justification for short 
segments only, like Kosambi's original formulæ they may often be found to apply in good approximation for larger segments. It will be seen that their form is consistent with the known disappearance of interference between long segments or widely separated segments.

It may be remarked in passing that the formulæ for 4 loci are adequate for the derivation of all formulæ required for 5 loci (4 segments) in virtue of identities of the type

$$
y_{12,3+4}=y_{123}+y_{124}-2 y_{1234} \text {. }
$$

In the present note I extend the results to give formulæ in $y, z$ and $x$ adequate to deal with as many as 6 loci ( 5 segments), and show how the formulæ may be adapted to apply to cases in which any or all of the pairs of segments are non-adjacent.

\section{FORMULA}

(a) 4 loci ( 3 adjacent segments).-

$$
\begin{aligned}
& y_{12}=y_{1} y_{2}-z_{1} z_{2}, \quad y_{23}=y_{2} y_{3}-z_{2} z_{3}, \\
& y_{13}=y_{1} y_{3}-z_{1} z_{3} e^{-4 x_{2}}, \\
& y_{123}=y_{1} y_{2} y_{3}-y_{1} z_{2} z_{3}-y_{2} z_{1} z_{3}-y_{3} z_{1} z_{2}+\frac{1}{2} z_{1} z_{3}\left(1-e^{-4 x_{2}}\right) .
\end{aligned}
$$

(b) 5 loci (4 adjacent segments).-Only one formula is essentially new, namely that for $y_{1234}$, all the others resulting either directly or by mere change of variables from those for 4 loci. However, it is instructive to write out the full set.

$$
\begin{aligned}
y_{12} & =y_{1} y_{2}-z_{1} z_{2}, \quad y_{14}=y_{1} y_{4}-z_{1} z_{4} e^{-4\left(x_{2}+x_{3}\right.} \\
y_{23} & =y_{2} y_{3}-z_{2} z_{3}, \quad y_{24}=y_{2} y_{4}-z_{2} z_{4} e^{-4 x_{3}} \\
y_{13} & =y_{1} y_{3}-z_{1} z_{3} e^{-4 x_{2}}, \quad y_{34}=y_{3} y_{4}-z_{3} z_{4}, \\
y_{123} & =y_{1} y_{2} y_{3}-y_{1} z_{2} z_{3}-y_{2} z_{1} z_{3}-y_{3} z_{1} z_{2}+\frac{1}{2} z_{1} z_{3}\left(\mathrm{I}-e^{-4 x_{2}}\right), \\
y_{124} & =y_{1} y_{2} y_{4}-y_{1} z_{2} z_{4} e^{-2 x_{3}}-y_{1} z_{1} z_{4} e^{-2 x_{3}}-y_{4} z_{1} z_{2}+\frac{1}{2} z_{1} z_{4}\left(\mathrm{I}-e^{-4 x_{2}}\right) e^{-4 x_{3}}, \\
y_{134} & =y_{1} y_{3} y_{4}-y_{1} z_{3} z_{4}-y_{3} z_{1} z_{4} e^{-2 x_{2}}-y_{4} z_{1} z_{3} e^{-2 x_{2}}+\frac{1}{2} z_{1} z_{4}\left(\mathrm{I}-e^{-4 x_{3}}\right) e^{-4 x_{2}}, \\
y_{234} & =y_{2} y_{3} y_{4}-y_{2} z_{3} z_{4}-y_{3} z_{2} z_{4}-y_{4} z_{2} z_{3}+\frac{1}{2} z_{2} z_{4}\left(\mathrm{I}-e^{-4 x_{2}}\right), \\
y_{1234} & =y_{1} y_{2} y_{3} y_{4}-y_{1} y_{2} z_{3} z_{4}-y_{2} y_{3} z_{1} z_{4}-y_{1} y_{3} z_{2} z_{4} \\
& -y_{1} y_{4} z_{2} z_{3}-y_{2} y_{4} z_{1} z_{3}-y_{2} y_{4} z_{1} z_{2}+z_{1} z_{2} z_{3} z_{4} \\
& +\frac{1}{2} y_{1} z_{2} z_{4}\left(\mathrm{I}-e^{-4 x_{3}}\right)+\frac{1}{2} y_{2} z_{1} z_{4}\left(\mathrm{I}-e^{-4 x_{3}}\right) \\
& +\frac{1}{2} y_{3} z_{1} z_{4}\left(\mathrm{I}-e^{-4 x_{3}}\right)+\frac{1}{2} y_{4} z_{1} z_{3}\left(\mathrm{I}-e^{-4 x_{2}}\right) \\
& -\frac{1}{4} z_{1} z_{4}\left(\mathrm{I}-e^{-4 x_{2}}\right)\left(\mathrm{I}-e^{-4 x_{3}}\right) .
\end{aligned}
$$

(c) 6 loci (5 segments).-Only two essentially new formulæ are required, namely :

$$
\begin{aligned}
y_{135}= & y_{1} y_{3} y_{5}-y_{1} z_{3} z_{5} e^{-4 x_{4}}-y_{3} z_{1} z_{5} e^{-4\left(x_{2}+x_{4}\right)} \\
& -y_{5} z_{1} z_{3} e^{-4 x_{2}}+\frac{1}{2} z_{1} z_{5}\left(1-e^{-4 x_{2}}\right) e^{-2\left(x_{2}+x_{4}\right)}
\end{aligned}
$$




$$
\begin{aligned}
& y_{12345}= y_{1} y_{2} y_{3} y_{4} y_{5}-\sum z_{1} z_{2} y_{3} y_{4} y_{5} \\
&-\frac{1}{2}\left\{z_{1} z_{5} y_{3} y_{4}\left(\mathrm{I}-e^{-4 x_{2}}\right)+z_{1} z_{5} y_{2} y_{4}\left(\mathrm{I}-e^{-4 x_{3}}\right)\right. \\
& \quad+z_{1} z_{5} y_{2} y_{3}\left(\mathrm{I}-e^{-4 x_{4}}\right)+z_{1} z_{3} y_{4} y_{5}\left(\mathrm{I}-e^{-4 x_{2}}\right) \\
& \quad+z_{1} z_{2} y_{4} y_{5}\left(\mathrm{I}-e^{-4 x_{3}}\right)+z_{1} z_{4} y_{3} y_{5}\left(\mathrm{I}-e^{-4 x_{2}}\right) \\
& \quad+z_{1} z_{4} y_{2} y_{5}\left(\mathrm{I}-e^{-4 x_{3}}\right)+z_{2} z_{5} y_{1} y_{3}\left(\mathrm{I}-e^{-4 x_{4}}\right) \\
&\left.\quad+z_{2} z_{5} y_{1} y_{4}\left(\mathrm{I}-e^{-4 x_{3}}\right)+z_{3} z_{5} y_{1} y_{2}\left(\mathrm{I}-e^{-4 x_{4}}\right)\right\} \\
&-\frac{1}{2}\left\{z_{1} z_{3} z_{4} z_{5}\left(\mathrm{I}-e^{-2 x_{2}}\right)+z_{1} z_{2} z_{4} z_{5}\left(\mathrm{I}-e^{-2 x_{3}}\right)\right. \\
&\left.\quad+z_{1} z_{2} z_{3} z_{5}\left(\mathrm{I}-e^{-2 x_{4}}\right)\right\} \\
&+\frac{1}{4}\left(z_{1} z_{5} y_{2}+z_{2} z_{5} y_{1}\right)\left(\mathrm{I}-e^{-4 x_{3}}\right)\left(\mathrm{I}-e^{-4 x_{4}}\right) \\
&+\frac{1}{4}\left(z_{1} z_{5} y_{3}+z_{3} z_{5} y_{1}\right)\left(\mathrm{I}-e^{-4 x_{4}}\right)\left(\mathrm{I}-e^{-4 x_{2}}\right) \\
&+\frac{1}{4}\left(z_{1} z_{5} y_{4}+z_{4} z_{5} y_{1}\right)\left(\mathrm{I}-e^{-4 x_{2}}\right)\left(\mathrm{I}-e^{-4 x_{3}}\right) \\
&-\frac{1}{8} z_{1} z_{5}\left(\mathrm{I}-e^{-4 x_{2}}\right)\left(\mathrm{I}-e^{-4 x_{3}}\right)\left(\mathrm{I}-e^{-4 x_{4}}\right) .
\end{aligned}
$$

(d) Extension of formule to separated segments.- It is easy to generalise any formula relating to contiguous segments so that it applies to non-adjacent ones. For example, the expression for $y_{1357}$ relating to intervals $x_{1}, x_{3}, x_{5}, x_{7}$ out of the contiguous set $x_{1}, x_{2}, x_{3}, x_{4}, x_{5}, x_{6}, x_{7}$, can be derived as follows :

If $x_{2}, x_{4}, x_{6}$ were all zero so that I, 3,5 and 7 were contiguous we would have

$$
\begin{aligned}
y_{1357}=y_{1} y_{3} y_{5} y_{7} & -z_{1} z_{3} y_{5} y_{7}-z_{1} z_{5} y_{3} y_{7}-z_{1} z_{7} y_{3} y_{5} \\
& -z_{3} z_{5} y_{1} y_{7}-z_{3} z_{7} y_{1} y_{5}-z_{5} z_{7} y_{1} y_{3} \\
+z_{1} z_{3} z_{5} z_{7} & +\frac{1}{2} z_{1} z_{7} y_{5}\left(\mathrm{I}-e^{-4 x_{3}}\right)+\frac{1}{2} z_{1} z_{7} y_{3}\left(\mathrm{I}-e^{-4 x_{5}}\right) \\
& +\frac{1}{2} z_{1} z_{5} y_{7}\left(\mathrm{I}-e^{-4 x_{3}}\right)+\frac{1}{2} z_{3} z_{7} y_{1}\left(\mathrm{I}-e^{-4 x_{5}}\right) \\
& -\frac{1}{4} z_{1} z_{7}\left(\mathrm{I}-e^{-4 x_{3}}\right)\left(\mathrm{I}-e^{-4 x_{6}}\right) .
\end{aligned}
$$

When $x_{2}, x_{4}, x_{6}$ are not zero this formula becomes

$$
\begin{aligned}
y_{1357}= & y_{1} y_{3} y_{5} y_{7}-z_{1} z_{3} y_{5} y_{7} e^{-4 x_{2}}-z_{1} z_{5} y_{3} y_{7} e^{-4\left(x_{2}+x_{4}\right)} \\
& -z_{1} z_{7} y_{3} y_{5} e^{-4\left(x_{8}+x_{4}+x_{6}\right)}-z_{3} z_{5} y_{1} y_{7} e^{-4 x_{4}} \\
& -z_{3} z_{7} y_{1} y_{5} e^{-4\left(x_{4}+x_{6}\right)}-z_{5} z_{7} y_{1} y_{3} e^{-4 x_{6}} \\
+ & z_{1} z_{3} z_{5} z_{7} e^{-4\left(x_{2}+x_{6}\right)} \\
+ & +\frac{1}{2} z_{1} z_{7} y_{5}\left(\mathrm{I}-e^{-4 x_{3}}\right) e^{-4\left(x_{4}+x_{4}+x_{6}\right)}+\frac{1}{2} z_{1} z_{7} y_{3}\left(\mathrm{I}-e^{-4 x_{5}}\right) e^{-4\left(x_{2}+x_{4}+x_{6}\right)} \\
+ & +\frac{1}{2} z_{1} z_{5} y_{7}\left(\mathrm{I}-e^{-4 x_{3}}\right) e^{-4\left(x_{3}+x_{4}\right)}+\frac{1}{2} z_{3} z_{7} y_{1}\left(\mathrm{I}-e^{-4 x_{5}}\right) e^{-4\left(x_{4}+x_{6}\right)} \\
& -\frac{1}{4} z_{1} z_{7}\left(\mathrm{I}-e^{-4 x_{3}}\right)\left(\mathrm{I}-e^{-4 x_{7}}\right) e^{-4\left(x_{4}+x_{4}+x_{6}\right)}
\end{aligned}
$$

The rule of derivation consists merely of inserting an attenuation factor corresponding to each term $z_{j} z_{k}$ as written and detemined by the total length of "gap intervals" between the segments $j$ and $k$. 


\section{METHOD OF PROOF OF THE FORMULE}

For $(k+\mathrm{r})$ loci $\left(k\right.$ contiguous segments $\left.x_{1}, x_{2}, \ldots, x_{k}\right)$ infinitely distant both from the centromere and the terminus of the chromosome arm we have a generating function (5) which takes the value

where

$$
\mathrm{P}\left(\lambda_{1}, \lambda_{2} \ldots, \lambda_{k}\right)=\frac{1}{2} \mathrm{Q} \exp \left(-\sum_{j=1}^{k} \mathrm{D}_{j}\right)
$$

$$
\begin{array}{r}
\text { and } \mathrm{Q}={ }_{2} \mathrm{C}_{1} \mathrm{C}_{2} \ldots \mathrm{C}_{k}+\Sigma \mathrm{S}_{1} \mathrm{C}_{2} \ldots \mathrm{C}_{k} \mathrm{~L}_{1}+\Sigma \mathrm{S}_{1} \mathrm{~S}_{2} \mathrm{C}_{3} \ldots \mathrm{C}_{k} \mathrm{~L}_{12}+\ldots \\
\quad \ldots+\mathrm{S}_{1} \mathrm{~S}_{2} \ldots \mathrm{S}_{k} \mathrm{~L}_{12} \ldots k \\
\text { with } \quad \mathrm{C}_{j}=\cosh 2 \sqrt{\lambda_{j}}, \quad \mathrm{~S}_{j}=\sinh 2 \sqrt{\lambda_{j}} .
\end{array}
$$

In a typical item such as $\mathrm{S}_{1} \mathrm{~S}_{2} \mathrm{C}_{3} \mathrm{~S}_{4} \mathrm{C}_{5} \mathrm{C}_{8} \mathrm{~S}_{7} \mathrm{C}_{8} \mathrm{~L}_{1247}$ the $\mathrm{L}$ symbol $\mathrm{L}_{1247}$ is to be read as the function

$$
\left(\frac{\sqrt{\lambda_{1}} \sqrt{\lambda_{4}}}{\sqrt{\lambda_{2}} \sqrt{\lambda_{7}}}+\frac{\sqrt{\lambda_{2}} \sqrt{\lambda_{7}}}{\sqrt{\bar{\lambda}_{1}} \sqrt{\lambda_{4}}}\right)
$$

Similarly $\mathrm{L}_{34568}$ in $\mathrm{C}_{1} \mathrm{C}_{2} \mathrm{~S}_{3} \mathrm{~S}_{4} \mathrm{~S}_{5} \mathrm{~S}_{6} \mathrm{C}_{7} \mathrm{~S}_{8} \mathrm{C}_{9} \mathrm{C}_{10} \mathrm{~L}_{34568}$ is

$$
\left(\frac{\sqrt{\lambda_{3}} \sqrt{\lambda_{5}} \sqrt{\lambda_{8}}}{\sqrt{\lambda_{4}} \sqrt{\bar{\lambda}_{6}}}+\frac{\sqrt{\lambda_{4}} \sqrt{\lambda_{8}}}{\sqrt{\bar{\lambda}_{3}} \sqrt{\lambda_{5}} \sqrt{\lambda_{8}}}\right)
$$

The multiple segmental recombination fractions can be calculated from the $2^{n}$ quantities obtained from $P$ by substituting values +1 or $-\mathrm{I}$ for the $\lambda$ 's (5). For example, if we set

$$
\lambda_{n_{\mathrm{t}}}=\lambda_{n_{\mathrm{s}}}=\ldots=\lambda_{n_{\mathrm{r}}}=-\mathrm{I}
$$

and all other $\lambda$ 's equal to $+\mathrm{r}$, the resulting value of $\mathrm{P}$ is equal to

$$
\mathrm{P}_{n_{1} n_{2} \ldots n_{r}}=\mathrm{I}-2 y_{n_{1}+n_{2}+\ldots n r}
$$

where $y_{n_{1}+n_{3}+\ldots+n_{r}}$ is the probability that the sum of the numbers of exchange points in the segments $x_{n_{1}}, x_{n_{z}}, \ldots, x_{n_{r}}$ will be odd.

It is easy to verify the elementary identity

$$
\mathrm{I}-2 y_{n_{1}+n_{2}+\ldots+n_{r}}=\mathrm{I}-2 \Sigma y_{n_{1}}+4 \Sigma y_{n_{1} n_{z}}-\ldots+(-)^{r} 2^{r} y_{n_{1} n_{1}} \ldots n_{r},
$$

where $y_{n_{1} n_{s}}$ is the probability that segments $x_{n_{1}}$ and $x_{n_{z}}$ will show simultaneous recombination, and so on. Thus the segmental recombination fractions $y_{n_{1} n_{2}}, y_{n_{1} n_{2} n_{3}}, \ldots, y_{12} \ldots k$ can be calculated inductively.

For example, with 3 adjacent segments we have

$$
\begin{aligned}
\mathrm{I}-2 & \left(y_{1}+y_{3}\right)+4 y_{13}=\mathrm{I}-2 y_{1+3}=\mathrm{P}_{13}=\mathrm{P}(-\mathrm{I}, \mathrm{I},-\mathrm{I}) \\
& =e^{-\left(\mathrm{D}_{1}+\mathrm{D}_{2}\right)}\left(\cos \mathrm{D}_{1} \cos \mathrm{D}_{3}-e^{-2 \mathrm{D}_{2}} \sin \mathrm{D}_{1} \sin \mathrm{D}_{3}\right) \\
& =\left(\mathrm{I}-2 y_{1}\right)\left(\mathrm{I}-2 y_{3}\right)-4 z_{1} z_{3} e^{-4 x_{2}} .
\end{aligned}
$$

Thus

$$
y_{13}=y_{1} y_{3}-z_{1} z_{3} e^{-4 x_{x}} \text {. }
$$


Similarly

$$
\begin{aligned}
& \mathrm{I}-2\left(y_{1}+y_{2}+y_{3}\right)+4\left(y_{12}+y_{23}+y_{13}\right)-8 y_{123} \\
& =\mathrm{I}-2 y_{1+2+3}=\mathrm{P}_{123}=\mathrm{P}(-\mathrm{I},-\mathrm{I},-\mathrm{I}) \\
& =e^{-\left(\mathrm{D}_{1}+\mathrm{D}_{2}+\mathrm{D}_{3}\right)}\left(\cos \mathrm{D}_{1} \cos \mathrm{D}_{2} \cos \mathrm{D}_{3}-\sin \mathrm{D}_{1} \sin \mathrm{D}_{2} \cos \mathrm{D}_{3}\right. \\
& \left.\quad-e^{-2 \mathrm{D}_{2}} \sin \mathrm{D}_{1} \cos \mathrm{D}_{2} \sin \mathrm{D}_{3}-\cos \mathrm{D}_{1}, \sin \mathrm{D}_{2} \sin \mathrm{D}_{3}\right)
\end{aligned}
$$

which ultimately gives the value of $y_{123}$.

\section{FORMULE IN TERMS OF RECOMBINATION FRACTIONS ONLY}

The substitutions

$$
\begin{aligned}
e^{-4 x_{r}} & \bumpeq\left(\mathrm{I}-2 y_{r}\right) /\left(\mathrm{I}+2 y_{r}\right) \\
z_{r} z_{s} & \bumpeq\left(\mathrm{I}-2 y_{r}\right)\left(\mathrm{I}-2 y_{s}\right) /\left(\mathrm{I}+4 y_{r} y_{s}\right)
\end{aligned}
$$

give formulæ generating those of Kosambi (I) and Owen (7). With $(k+1)$ linked loci they could be used for fitting the $k$ parameters $y_{1}, y_{2}, \ldots, y_{h}$ to observed recombination data by maximum likelihood scoring.

\section{THE AUXILIARY QUANTITY $z$}

$$
z=\frac{1}{2} e^{-2 x} \sin 2 x
$$

is the imaginary part of $w$ where

$$
\mathrm{I}-2 w=e^{-2 x(1+i)},
$$

so that $w=y+i z$ satisfies Trow's formula for adjacent segments at mid-arm.

i.e.

$$
w_{1+2}=w_{1}+w_{2}-2 w_{1} w_{2}
$$

Thus the effect of interference is formally removed for adjacent segments by the use of $w$, which may be called the complex recombination fraction or "complexit", its components $y$ and $z$ being named respectively the "recombit" and the "cocombit".

For short segments we have the expansions

$$
y=\frac{1}{2}\left(1-e^{-2 x} \cos 2 x\right)=x-\frac{4}{3} x^{3}+\ldots
$$

as in Kosambi's formula.

$$
\begin{aligned}
z & =\frac{1}{2} e^{-2 x} \sin 2 x=x-2 x^{2}+\frac{4}{3} x^{3}+\ldots \\
x & =y+\frac{4}{3} y^{3}+\ldots \\
z & =y-2 y^{2}+\frac{8}{3} y^{3}+\ldots \\
& =y(\mathrm{I}-2 y) /\left(\mathrm{I}-\frac{8}{3} y^{2}\right)+o\left(y^{4}\right) .
\end{aligned}
$$

Therefore

The second order approximation to the cocombit is therefore $y(1-2 y)$, but its use is rather less effective than that of the approximation for $z_{r} z_{s}$. It may be noted, however, that a good second order approxima- 
tion is provided by $y(\mathrm{I}-\mathrm{I} \cdot 56 y)$. The following table gives percentage values of $x(c \mathrm{M})$, of $y$ and of $\frac{1}{2} \tanh 2 x$ and also of $z$ and of $y(\mathrm{I}-\mathrm{I} \cdot 56 y)$.

\begin{tabular}{|c|c|c|c|c|}
\hline$\underset{c \stackrel{x}{M}}{x}$ & $\frac{1}{2} \tanh 2 x$ & $y$ & $z$ & $y(\mathrm{I}-\mathrm{I} \cdot 56 y)$ \\
\hline 0 & 0 & 0 & 0 & 0 \\
\hline 5 & $4.9^{8} 4$ & 4.984 & $4 \cdot 517$ & $4 \cdot 597$ \\
\hline 10 & $9^{\circ} \cdot 869$ & 9.879 & $8 \cdot 133$ & $8 \cdot 357$ \\
\hline I 5 & 14.566 & 14.614 & $10.94^{6}$ & II $\cdot 282$ \\
\hline 20 & 18.998 & 19.130 & 13.052 & $\mathrm{I} 3.42 \mathrm{I}$ \\
\hline 25 & $23 \cdot 106$ & $23 \cdot 386$ & $14.54^{\circ}$ & 14.854 \\
\hline $3^{\circ}$ & $26 \cdot 85^{2}$ & $27 \cdot 35^{2}$ & I 5.494 & $15.68 \mathrm{I}$ \\
\hline 35 & $30 \cdot 218$ & 31.010 & I 5.995 & I $6 \cdot 009$ \\
\hline $4^{\circ}$ & $33 \cdot 202$ & $34 \cdot 34^{8}$ & $16 \cdot 116$ & I 5.943 \\
\hline 45 & $35 \cdot 815$ & $37 \cdot 36_{4}$ & 15.924 & 15.585 \\
\hline $5^{\circ}$ & $38 \cdot 080$ & 40.062 & I 5.478 & I 5.025 \\
\hline 55 & 40.025 & $4^{2} \cdot 45^{I}$ & I 4.833 & I 4.339 \\
\hline 60 & $4^{I} \cdot 683$ & 44.543 & 14.036 & I $3.59^{\mathrm{I}}$ \\
\hline 65 & $43 \cdot 086$ & $4^{6} \cdot 355$ & I $3 \cdot 130$ & I 2.834 \\
\hline 70 & $44 \cdot 268$ & 47.904 & I 2.150 & I2.105 \\
\hline 75 & 45.257 & 49.211 & II $\cdot$ I 29 & II $\cdot 43^{2}$ \\
\hline 80 & $46 \cdot 084$ & 50.029 & $10.09 \mathrm{I}$ & 10.983 \\
\hline 85 & $46 \cdot 770$ & $5 \mathrm{I} \cdot \mathrm{I} 75$ & $9 \cdot 05^{8}$ & $10 \cdot 320$ \\
\hline 90 & $47 \cdot 34^{\circ}$ & $5 \mathrm{I} \cdot 894$ & $8 \cdot 049$ & $9 \cdot 883$ \\
\hline 95 & $47 \cdot 8 \div 2$ & $52 \cdot 462$ & 7.077 & 9.527 \\
\hline 100 & $4^{8 \cdot 201}$ & $52 \cdot 904$ & $6 \cdot 153$ & $9 \cdot 24^{2}$ \\
\hline
\end{tabular}

\section{LOCI PROXIMAL TO CENTROMERE ON A LONG ARM}

It is easy to derive results applying to segments on a long arm which are at finite distances from the centromere. We replace the map distance $x$, wherever it appears in the existing formulæ, by the interference metric $t$ and treat the centromere as having the properties of a localised exchange point $(4,5)$.

Consider 3 adjacent segments $\mathrm{O}, \mathrm{I}, 2$ which have the metrical specification $\left(0, t_{0}\right),\left(t_{0}, t_{1}\right),\left(t_{1}, t_{2}\right)$. We have

where

$$
y_{02}=y_{0} \eta_{2}-z_{0} \zeta_{2} e^{-4 D_{1}}
$$

$$
\eta=\frac{1}{2}\left(\mathrm{I}-e^{-2 x} \cos 2 \mathrm{D}\right), \zeta=\frac{1}{2} e^{-2 \mathrm{D}} \sin 2 \mathrm{D}
$$

and $\mathrm{D}$ is now metrical length, i.e. $\mathrm{D}_{1}=\left(t_{1}-t_{0}\right)$.

The probability of recombination in 2 conditional on recombination in $o$ is

$$
y_{02} / y_{0}=\eta_{2}-e^{-4 \mathrm{D}_{1}} \zeta_{2}\left(z_{0} / y_{0}\right) \text {. }
$$

The limit of this probability as $t_{0} \rightarrow 0$ is clearly the probability of recombination in 2 conditional on there being an exchange point at $t=0$, and therefore gives the recombination over a segment $\left(t_{1}, t_{2}\right)$ at metrical distance $t_{1}$ from the centromere as

$$
y_{2}=\eta_{2}-e^{-4 t_{1}} \zeta_{2}
$$

as given by Owen (4). 
Similarly for simultaneous recombination in separated intervals we have

$$
y_{13}=\eta_{1} \eta_{3}-\zeta_{1} \zeta_{3} e^{-4 D_{2}}-e^{-4 t_{1}}\left\{\zeta_{1} \eta_{3}+\zeta_{3} \eta_{1} e^{-4 D_{2}}-\frac{1}{2} \zeta_{3}\left(\mathrm{I}-e^{-4 D_{1}}\right) e^{-4 D_{5}}\right\} .
$$

Also

$$
\begin{aligned}
y_{123}=\eta_{1} \eta_{2} \eta_{3}- & \left(\eta_{1} \zeta_{2} \zeta_{3}+\eta_{2} \zeta_{1} \zeta_{3}+\eta_{3} \zeta_{1} \zeta_{2}\right)+\frac{1}{2} \zeta_{1} \zeta_{3}\left(\mathrm{I}-e^{-4 D_{2}}\right) \\
-e^{-4 t_{1}} & \left\{\eta_{1} \eta_{2} \zeta_{3}+\eta_{2} \eta_{3} \zeta_{1}+\eta_{1} \eta_{3} \zeta_{2}-\zeta_{1} \zeta_{2} \zeta_{3}\right. \\
& -\frac{1}{2} \eta_{1} \zeta_{3}\left(\mathrm{I}-e^{-4 D_{2}}\right)-\frac{1}{2} \eta_{2} \zeta_{3}\left(\mathrm{I}-e^{-4 D_{1}}\right) \\
& \left.-\frac{1}{2} \zeta_{2} \eta_{3}\left(\mathrm{I}-e^{-4 D_{1}}\right)-\frac{1}{4} \zeta_{3}\left(\mathrm{I}-e^{-4 D_{1}}\right)\left(\mathrm{I}-e^{-4 \mathbf{D}_{2}}\right)\right\}
\end{aligned}
$$

\section{SUMMARY}

Generalisations of Kosambi's formulæ are obtained serving to give the frequencies of multiple recombination in as many as 5 adjacent or separated segments. The formulæ are expressed in terms of recombination fractions for single segments and auxiliary quantities $z$. It is shown how to obtain complete generalisations of Kosambi's formulæ, expressed purely in terms of the recombination fractions.

\section{ELEMENTARY FORMULA FOR MULTIPLE RECOMBINATION OF CLOSELY LINKED LOCI}

\section{INTRODUCTION}

It has been seen that for contiguous segments I and 2 Kosambi (I) gives the useful addition formula

$$
y_{1+2}=\left(y_{1}+y_{2}\right) /\left(\mathrm{I}+4 y_{1} y_{2}\right)
$$

connecting the recombination fractions $y_{1}, y_{2}$ and $y_{1+2}$ in the segments $\mathrm{I}$ and 2 and the joint segment $\mathrm{I}+2$. This has been found to be substantially correct for long segments in the mouse ( $\mathrm{IO}$ ), in rice (II), and in the sweet pea (12) and to hold for loci situated medianly in the arms of chromosomes VI and III in Drosophila melanogaster (2). Theoretical reasons have been advanced why this should be so $(5,7)$. by

Since the frequency of simultaneous recombination is given always

$$
y_{12}=\frac{1}{2}\left(y_{1}+y_{2}-y_{1+2}\right)
$$

it follows from Kosambi's formula that the frequency of doubles is given by

$$
y_{12}=2 y_{1} y_{2}\left(y_{1}+y_{2}\right) /\left(\mathrm{I}+4 y_{1} y_{2}\right)
$$

It was shown above that for non-contiguous segments an appropriate generalisation of this result is

$$
y_{13}=y_{1} y_{3}\left\{\mathrm{I}-\frac{\mathrm{I}-2 y_{2}}{\mathrm{I}+2 y_{2}} \frac{\left(\mathrm{I}-2 y_{1}\right)\left(\mathrm{I}-2 y_{3}\right)}{\mathrm{I}+4 y_{1} y_{3}}\right\} .
$$


For short segments when $y_{1}, y_{2}, y_{3}$ are small, if we retain only terms of the third order we get the formulæ

$$
y_{12}=2 y_{1} y_{2}\left(y_{1}+y_{2}\right), \quad y_{13}=2 y_{1} y_{3}\left(y_{1}+2 y_{2}+y_{3}\right) \text {. }
$$

Similar formulæ could be got for the frequencies of triple, quadruple crossing over, etc. using the previous results. However, Professor Sir Ronald Fisher suggested to me that they could be derived rather simply by a completely elementary method, which may be illustrated by the case of 4 linked loci ( 3 contiguous segments).

We note that if segment 3 is so short that $y_{3}$ may be replaced by the differential $d y$ then the probability of simultaneous recombination in segment $\mathrm{I}$ and the segment $d y$ is

$$
y_{13}=2 y_{1}\left(y_{1}+2 y_{2}\right) d y
$$

Equation (I) also implies that the probability of recombination in I conditional on recombination in $d y$ is

$$
y_{13} / d y=2 y_{1}\left(y_{1}+2 y_{2}\right) \quad . \quad . \quad .
$$

Results (I) and (2) enable the frequencies of simultaneous recombination in any number of short contiguous segments to be calculated inductively provided that a further simple assumption is made. This assumption is in accordance with recombination theory as previously developed. We suppose that a cross-over point once established effectively divides the chromosome into non-interfering regions.

Let now $p, q, r$ be the frequencies of recombination in contiguous segments $\mathrm{AB}, \mathrm{BC}, \mathrm{CD}$ supposed short but not infinitesimal. Consider an elementary segment $d y$ located at a point $\mathrm{P}$ interior to $\mathrm{BC}$. Then to the present approximation the recombination over $\mathrm{BP}$ is $y$ and over PC is $(q-y)$. By (I) the probability of simultaneous recombination in $\mathrm{AB}$ and $d y$ is

$$
2 p(p+2 y) d y \text {. }
$$

But by (2) the probability of recombination in CD conditional on recombination in $d y$ is

$$
2 r(r+2 \overline{q-y}) .
$$

Thus the probability of recombination simultaneously in $A B, d y$ and $\mathrm{CD}$ is

$$
4 p r(p+2 y)(r+2 q-2 y) d y .
$$

It is now easy to see that, on account of the assumed smallness of $p, q$ and $r$, the probability of simultaneous recombination $y_{12}$ in $\mathrm{AB}, \mathrm{BC}$ and $\mathrm{CD}$ is merely

which is

$$
\int_{0}^{q} 4 p r(p+2 y)(r+2 q-2 y) d y
$$

$$
4 p r\left\{(p+q)(q+r)-\frac{1}{3} q^{2}\right\}
$$


The results of this method are assembled for 4,5 and 6 loci in section 3. It will be seen that a parameter $\mathrm{K}$ has been introduced. This will be discussed below.

\section{THE KOSAMBI COEFFICIENT}

The Kosambi coefficient $\mathrm{K}$ for three linked loci is defined as

$$
\mathrm{K}=\frac{y_{1}+y_{2}-y_{1+2}}{4 y_{1} y_{2} y_{1+2}}=\frac{y_{12}}{2 y_{1} y_{2} y_{1+2}} .
$$

The value of $\mathrm{K}$ is in general different from unity. $\mathrm{K}$ is equal to unity, if and only if, Kosambi's formula holds exactly. The value of $\mathrm{K}$ as estimated from 3 point data may be used to test agreement with Kosambi's formula. When $\mathrm{K}$ is significantly less than unity then the genetical interference which is operating must be concluded to be more intense than that predicted by Kosambi's formula. The opposite conclusion is indicated by an observed $\mathrm{K}$ significantly exceeding unity. Reasons have been given (6) for supposing that in regions near the centromere $\mathrm{K}$ is large, and thereafter diminishes. steadily becoming less than unity in regions near the terminus of a chromosome arm. At some intermediate region (actually near metrical mid-arm) $\mathrm{K}$ is approximately unity and Kosambi's formula will apply there. These expectations are confirmed by the data on Chromosomes II and III of Drosophila melanogaster (2). Thus an observed value of $\mathrm{K}$ is some indication of the location of the triplet of loci on the chromosome arm.

Inclusion of the parameter $\mathrm{K}$ modifies the formulæ for short segments in a simple way and confers generality upon them.

If $\mathrm{K}$ is defined as above then we have identically for the frequency of doubles

$$
y_{12}={ }_{2} \mathrm{~K} y_{1} y_{2} y_{1+2}={ }_{2} \mathrm{~K} y_{1} y_{2}\left(y_{1}+y_{2}\right) /\left(\mathrm{I}+{ }_{4} \mathrm{~K} y_{1} y_{2}\right),
$$

which for short segments is effectively

$$
y_{12}=2 \mathrm{~K} y_{1} y_{2}\left(y_{1}+y_{2}\right)={ }_{2} \mathrm{~K} y_{1}\left(y_{1} y_{2}+y_{2}{ }^{2}\right)
$$

Now suppose that the segment $y_{2}$ is increased to be $y_{2}+d y$ by the addition of an elementary segment $d y$. Then the resulting increase in $y_{12}$ is given by

$$
d y_{12}={ }_{2} \mathrm{~K} y_{1}\left(y_{1}+2 y_{2}\right) d y+2 y_{1} y_{2}\left(y_{1}+y_{2}\right) \frac{\partial \mathrm{K}}{\partial y_{2}} d y
$$

The second term is of the third degree in $y_{1}, y_{2}$ and consequently may be neglected in the present approximation. Thus

$$
d y_{12}=2 \mathrm{~K} y_{1}\left(y_{1}+2 y_{2}\right) d y
$$

and the probability of simultaneous recombination in $\mathrm{AB}$ and $d y$ becomes

$$
{ }_{2} \mathrm{~K} p(p+2 y) d y,
$$

$\mathrm{K}$ appearing merely as a multiplier. 
To the present order of accuracy therefore, $\mathrm{K}$ is to be regarded as a constant over the total region occupied by the segments and the formulæ are merely multiplied by powers of $\mathrm{K}$.

\section{FORMULA}

(a) Notation.- $p, q, r, s, t$, denote contiguous segments taken in order. It is convenient to define the following symbols.

$$
\begin{aligned}
(p, q, r) & =(p+q)(q+r)-\frac{1}{3} q^{2} \\
(p, q, r, s) & =(p+q)(q+r)(r+s)-\frac{1}{3} q^{2}(r+s)-\frac{1}{3} r^{2}(p+q) \\
(p, q, r, s, t) & =(p, q, r)(r, s, t)-\frac{1}{3} r^{2}(p+q)(r+t)
\end{aligned}
$$

(b) 4 loci (3 segments).-

$$
\begin{array}{rlr}
y_{12} & =2 \mathrm{~K} p q(p+q), & \\
y_{13} & =2 \mathrm{~K} p r(p+2 q+r) & \\
y_{123} & =4 \mathrm{~K}^{2} p q r(p, q, r) &
\end{array}
$$

(c) 5 loci (4 segments).--

$$
\begin{array}{rlrl}
y_{12} & =2 \mathrm{~K} p q(p+q), & & y_{13}=2 \mathrm{~K} p r(p+2 q+r) \\
y_{14} & =2 \mathrm{~K} p s(p+2 q+r+s), & & y_{23}=2 \mathrm{~K} q r(q+r), \\
y_{24} & =2 \mathrm{~K} q s(q+s), & & y_{34}=2 \mathrm{~K} r s(r+s), \\
y_{234} & ={ }_{4} \mathrm{~K}^{2} q r s(q, r, s), & y_{123}=4 \mathrm{~K}^{2} p q r(p, q, r), \\
y_{124} & =4 \mathrm{~K}^{2} p q s\left\{(p+q)(q+2 r+s)-\frac{1}{3} q^{2}\right\}, \\
y_{134} & =4 \mathrm{~K}^{2} p r s\left\{(r+s)(p+2 q+r)-\frac{1}{3} r^{2}\right\}, \\
y_{1234} & =8 \mathrm{~K}^{3} p q r s(p, q, r, s) . &
\end{array}
$$

It will be seen that with the exception of the last three, these expressions result mutatis mutandi from the appropriate 3-point formulæ. For instance $y_{14}$ is derived from $2 \mathrm{~K} p r(p+2 q+r)$ by the substitutions $s$ for $r, q+r$ for $q(q+r$ being to the present degree of approximation the recombination in the separating segment $(2+3))$. The formulæ for $y_{124}$ and $y_{134}$ contain nothing essentially new being calculated from the relations

$$
y_{124}=y_{123+4}-y_{123}, \quad y_{134}=y_{1+234}-y_{234},
$$

which hold in the present approximation. Their form shows them however to be derivable by a simple rule. We may write

$$
y_{124}={ }_{4} \mathrm{~K}^{2} p q s(p, q, s)^{1}
$$

where $(p, q, s)^{1}$ results from $(p, q, s)$ by inserting in the item $(q+s)$ the term $2 r$ corresponding to the separating segment $r$.

(d) 6 loci ( 5 segments).-Formulæ for two segments such as

$$
y_{15}=2 \mathrm{~K} p t(p+2 q+2 r+2 s+t)
$$

follow trivially from the previous results. 
The formulæ for three or four segments follow either directly or by application of the rule for separating segments, e.g.

$$
\begin{aligned}
y_{125} & =4 \mathrm{~K}^{2} p q t(p, q, t)^{1}={ }_{4} \mathrm{~K}^{2} p q t\left\{(p+q)(q+2 r+2 s+t)-\frac{1}{3} q^{2}\right\}, \\
y_{135} & ={ }^{2} \mathrm{~K}^{2} p r t(p, r, t)^{\mathbf{1}}={ }_{4} \mathrm{~K}^{2} p r t\left\{(p+2 q+r)(r+2 s+t)-\frac{1}{3} r^{2}\right\}, \\
y_{1235} & =8 \mathrm{~K}^{3} p q r t(p, q, r, t)^{1} \\
& =8 \mathrm{~K}^{3} p q r t\left\{(p+q)(q+r)(r+2 s+t)-\frac{1}{3} q^{2}(r+2 s+t)-\frac{1}{3} r^{2}(p+q)\right\}, \\
y_{1245} & =8 \mathrm{~K}^{3} p q s t(p, q, s, t)^{1} \\
& =8 \mathrm{~K}^{3} p q s t\left\{(p+q)(q+2 r+s)(s+t)-\frac{1}{3} q^{2}(s+t)-\frac{1}{3} s^{2}(p+q)\right\} .
\end{aligned}
$$

The formula for 5 segments is essentially new and can be found only by integration.

$$
y_{12345}=\mathrm{I}_{6} 6 \mathrm{~K}^{4} p q r s t(p, q, r, s, t)
$$

\section{VERIFICATION}

The expression

$$
y_{123}=y_{1} y_{2} y_{3}-y_{1} z_{2} z_{3}-y_{2} z_{1} z_{3}-y_{3} z_{1} z_{2}+\frac{1}{2} z_{1} z_{3}\left(1-e^{-4 x_{2}}\right),
$$

given previously (loc. cit.) reduces to the form $4 p q r(p, q, r)$ on putting

$$
\begin{aligned}
z & =y-2 y^{2}+\frac{8}{3} y^{3}, \\
e^{-4 x} & =\mathrm{I}-4 y+8 y^{2}-\mathrm{I} 6 y^{3}+\ldots=(\mathrm{I}-2 y)(\mathrm{I}+2 y) \\
& =\exp \left\{-4\left(y+\frac{8}{3} y^{3}\right)\right\}+\mathrm{o}\left(y^{4}\right),
\end{aligned}
$$

and retaining only terms of the fifth degree. This serves as a mutual check of both formulæ.

A similar comparison may be made with the generalised Kosambi formula given by the writer (loc cit.) namely

$$
y_{123}=p y_{23}-q y_{13}+r y_{12}
$$

with $y_{12}, y_{13}$ given by Kosambi's formula and

$$
y_{13}=p r\left\{\mathrm{I}-\frac{\mathrm{I}-2 q}{\mathrm{I}+2 q} \frac{(\mathrm{I}-2 p)(\mathrm{I}-2 r)}{\mathrm{I}+4 p r}\right\} \quad .
$$

With small segments we have correct to the $5^{\text {th }}$ degree

$$
y_{123}=4 p q r .2 q(p+q+r) \text {. }
$$

Consequently the generalised Kosambi formula implies rather a higher frequency of triples (about $5^{\circ}$ per cent. more). This is because it is essentially a quadratic approximation and not a cubic one.

\section{SUMMARY}

An elementary method is developed by which the frequencies of simultaneous recombination of several closely linked loci can be specified, and formulæ are given for as many as 6 such loci. 


\section{REFERENCES}

(I) KOSAMBI, D. D. 1944. The estimation of map distances from recombination values. Ann. Eugen., 12, I 72-1 75 .

(2) owen, A. R. G. 1948. Ph.D. Dissertation, pp. 204-219. Cambridge : University Library.

(3) FISHER, R. A., LYON, M. F., AND OWEN, A. R. G. 1947. The sex chromosome in the house mouse. Heredity, $1,355-365$.

(4) OWEN, A. R. G. 1949. The theory of genetical recombination. I. Longchromosome arms. P.R.S., Lond., B, 136, 67-94.

(5) OWEN, A. R. G. 1950. The theory of genetical recombination. Advances in Genetics, 3, I 1 7-157.

(6) FISHER, R. A. I95I. A combinatorial formulation of multiple linkage tests. Nature, 167,520 .

(7) OWEN, A. R. G. 1951. An extension of Kosambi's formula. Nature, 168, 208.

(8) stevens, w. L. 1936. The analysis of interference. 3. Genet., 32, 51-64.

(9) FISHER, R. A. I950. Statistical Methods for Research Workers, i ith Ed., p. 309.

(10) BORGER, R. 1950. The order of genes in the fifth linkage group of the house mouse. Nature, $166,197$.

(I I) BHAT, N. R. 1950. A case in rice, Oryza sativa L., supporting Kosambi's formula for estimating map lengths. Amer. Nat., 84, $71-80$.

(12) Bнat, N. R. 1948. An improved genetical map of Punnett's " B" chromosome in the Sweet Pea, Lathyrus odoratus L. 7. Genet., 48, 343-348. 